MАТНЕМАТІСА, 61 (84), $\mathrm{N}^{\circ}$ 1, 2019, pp. 22-32

\title{
NEW INTEGRAL RESULTS ON HOLDER TYPE INEQUALITIES
}

\author{
ABDELKADER BENZIDANE, HATICE YALDIZ, and ZOUBIR DAHMANI
}

\begin{abstract}
In this paper, using fractional integration, we present new fractional integral inequalities related to Holder inequality. We generalise a Wu's sharpness of Holder inequality for $p, q$ integration. Then, as an application, we propose another way to derive the Holder inequality which is already established by Z Dahmani on 2012 in General Math. Journal. Also, for our results, the classical Holder inequality is deduced as a special case.
\end{abstract}

MSC 2010. 26D15, 26A33, 60E15.

Key words. Integral inequality, Holder inequality, Riemann-Liouville integral.

\section{REFERENCES}

[1] S. Belarbi and Z. Dahmani, On some new fractional intégral inequalities, Journal of Inequalities in Pure and Applied Mathematics, 10 (2009), Article 86, 1-5.

[2] Z. Dahmani, New inequalities in fractional integrals, International Journal of Nonlinear Sciences, 9 (2010), 493-497.

[3] Z. Dahmani, About some integral inequalities using Riemann-Liouville integrals, Gen. Math., 20 (2012), 63-69.

[4] Z. Dahmani, O. Mechouar and S. Brahami, Certain inequalities related to the Chebyshev's functional involving Riemann-Liouville operator, Bulletin of Mathematical Analysis and Applications, 3 (2011), 38-44.

[5] Z. Dahmani and L. Tabharit, On weighted Gruss type inequalities via fractional integrals, Journal of Advanced Research in Pure Mathematics, 2 (2010), 31-38.

[6] S.S. Dragomir, A generalization of Gruss inequality in inner product spaces and applications, J. Math. Anal. Appl., 237 (1999), 74-82.

[7] R. Gorenflo and F. Mainardi, Fractional calculus: integral and differential equations of fractional order, Springer Verlag, Wien, 1997, 223-276.

[8] G. Hardy, J.E. Littlewood, G. Pólya, Inequalities, Second Ed., Cambridge University Press, UK, 1952.

[9] M.Z. Sarikaya and H. Yaldiz, New generalization fractional inequalities of OstrowskiGruss type, Lobachevskii J. Math., 34 (2013), 326-331.

[10] M.Z. Sarikaya, N. Aktan and H. Yildirim, On weighted Chebyshev-Gruss like inequalities on time scales, J. Math. Inequal., 2 (2008), 185-195.

[11] S.H. Wu, Generalization of a sharp Holder's inequality and its application, J. Math. Anal. Appl., 332 (2007), 741-750.

Received March 31, 2018

Accepted September 11, 2018

DOI: $10.24193 /$ mathcluj.2019.1.02 
UMAB University of Mostaganem Faculty of SEI

Department of Mathematics Algeria

E-mail: aekm27@yahoo.fr

Karamanoğlu Mehmetbey University Kamil Özdă̆ Science Faculty

Department of Mathematics Karaman, Turkey

E-mail: yaldizhatice@gmail.com

UMAB University of Mostaganem

Laboratory LPAM

Algeria

E-mail: zzdahmani@yahoo.fr 